\title{
Binary Pictures with Excluded Patterns
}

\author{
Daniela Battaglino ${ }^{1}$, Andrea Frosini ${ }^{2}$, Veronica Guerrini ${ }^{1}$, \\ Simone Rinaldi ${ }^{1}$, and Samanta Socci ${ }^{1}$ \\ 1 Università di Siena, Dipartimento di Matematica e Informatica, \\ Pian dei Mantellini 44, 53100 Siena \\ 2 Università di Firenze, Dipartimento di Sistemi e Informatica, \\ viale Morgagni 65, 50134 Firenze
}

\begin{abstract}
The notion of a pattern within a binary picture (polyomino) has been introduced and studied in [3], and resembles the notion of pattern containment within permutations. The main goal of this paper is to extend the studies of [3] by adopting a more geometrical approach: we use the notion of pattern avoidance in order to recognize or describe families of polyominoes defined by means of geometrical constraints or combinatorial properties. Moreover, we extend the notion of pattern in a polyomino, by introducing generalized polyomino patterns, so that to be able to describe more families of polyominoes known in the literature.
\end{abstract}

\section{Patterns in Binary Pictures and Polyomino Classes}

In recent years a considerable interest in the study of the notion of pattern within a combinatorial structure has grown. This kind of research started with patterns in permutations [12, while in the last few years it is being carried on in several directions. One of them is to define and study analogues of the concept of pattern in permutations in other combinatorial objects such as set partitions [1114], words, trees [13. The works 34] fit into this research line, in particular [4] introduces and studies the notion of pattern in finite binary pictures (specifically, in polyominoes).

A finite binary picture is an $m \times n$ matrix of 0's and 1's. Intuitively speaking, 1 's correspond to black pixels (which constitute the image) and the 0's correspond to white pixels (which form the background). Often, the studied images should fulfill several additional properties like symmetry, connectivity, or convexity. In particular, an image is connected if the set of black pixels is connected with respect to the edge-adjacency relation. A connected image is usually called a polyomino (see Figure 1).

The work [3], from which we borrow most of the basic definitions and notations, uses an algebraic setting to provide a unified framework to describe and handle some families of binary pictures (in particular polyominoes), by the avoidance of patterns. Therefore, in order to fruitfully present our paper, we need to recall some definitions and the main results from [3].

Let $\mathfrak{M}$ be the class of binary pictures (or matrices). We denote by $\preccurlyeq$ the usual subpicture (or submatrix) order on $\mathfrak{M}$, i.e. $M^{\prime} \preccurlyeq M$ if $M^{\prime}$ may be obtained from $M$ by deleting any collection of rows and/or columns. 
(a)

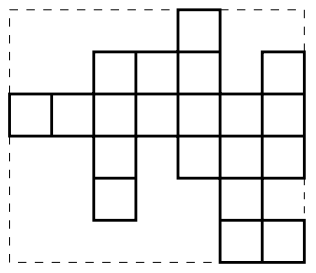

(b)

$\left[\begin{array}{lllllll}0 & 0 & 0 & 0 & 1 & 0 & 0 \\ 0 & 0 & 1 & 1 & 1 & 0 & 1 \\ 1 & 1 & 1 & 1 & 1 & 1 & 1 \\ 0 & 0 & 1 & 0 & 1 & 1 & 1 \\ 0 & 0 & 1 & 0 & 0 & 1 & 0 \\ 0 & 0 & 0 & 0 & 0 & 1 & 1\end{array}\right]$

Fig. 1. A polyomino and its representation as a binary picture (or matrix)

Notice that, in a binary picture representing a polyomino the first (resp. the last) row (resp. column) should contain at least a 1 . We can consider the restriction of the submatrix order $\preccurlyeq$ on the set of polyominoes $\mathfrak{P}$. This defines the poset $\left(\mathfrak{P}, \preccurlyeq_{P}\right)$ and the pattern order between polyominoes: a polyomino $P$ is a pattern of a polyomino $Q$ (which we denote $P \preccurlyeq_{P} Q$ ) when the binary picture representing $P$ is a submatrix of that representing $Q$. We point out that the order $\preccurlyeq P$ has already been studied in [8] under the name of subpicture order, where the authors - among other things - proved that $\left(\mathfrak{P}, \preccurlyeq_{P}\right)$ contains infinite antichains, and it is a graded poset (the rank function being the semi-perimeter of the bounding box of the polyominoes).

This allows to introduce a natural analogue of permutation classes for polyominoes: a polyomino class is a set of polyominoes $\mathcal{C}$ that is downward closed for $\preccurlyeq_{P}$ : for all polyominoes $P$ and $Q$, if $P \in \mathcal{C}$ and $Q \preccurlyeq_{P} P$, then $Q \in \mathcal{C}$. Basing on the results obtained in [1, in [3] the authors proved that some of the most famous families of polyominoes, including: the bargraphs, the convex, the column-convex, the L-convex, the directed-convex polyominoes, are indeed polyomino classes. On the other side, there are also well-known families of polyominoes which are not polyomino classes, like: the family of polyominoes having a square shape, the family of polyominoes having exactly $k>1$ columns, or the directed polyominoes (see Section 4).

Similarly to the case of permutations, for any set $\mathcal{B}$ of polyominoes, let us denote by $A v_{P}(\mathcal{B})$ the set of all polyominoes that do not contain any element of $\mathcal{B}$ as a pattern. Every such set $A v_{P}(\mathcal{B})$ of polyominoes defined by pattern avoidance is a polyomino class. Conversely, like for permutation classes, every polyomino class may be characterized in this way [3].

Proposition 1. For every polyomino class $\mathcal{C}$, there is a unique antichain $\mathcal{B}$ of polyominoes such that $\mathcal{C}=A v_{P}(\mathcal{B})$. The set $\mathcal{B}$ consists of all minimal polyominoes (in the sense of $\preccurlyeq_{P}$ ) that do not belong to $\mathcal{C}$.

We call $\mathcal{B}$ the polyomino-basis (or -basis for short), to distinguish from other kinds of bases. We observe that, denoting $A v_{M}(\mathcal{M})$ the set of binary matrices that do not have any submatrix in $\mathcal{M}$, we have $A v_{P}(\mathcal{M})=A v_{M}(\mathcal{M}) \cap \mathfrak{P}$.

On the other side, it is quite natural to describe classes of polyominoes by the avoidance of submatrices, then we introduce the notion of matrix-basis (or 
$m$-basis ) of a polyomino class $\mathcal{C}$, which is every antichain $\mathcal{M}$ of matrices such that $\mathcal{C}=A v_{P}(\mathcal{M})$. Differently from the $p$-basis, the $m$-basis needs not be unique.

Example 1 (Injections). Let $\mathcal{I}$ be the class of injections, i.e. polyominoes having at most a zero entry for each row and column such as, for instance
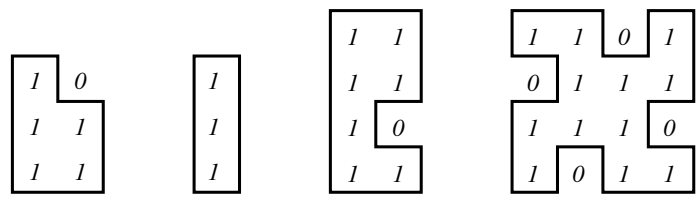

The set $\mathcal{I}$ is clearly a polyomino class, and its $p$-basis is given by the minimal polyominoes which are not injections, i.e. the twelve polyominoes on the top of Fig. 2. An $m$-basis of $\mathcal{I}$ is clearly given by set

$$
\mathcal{M}=\left\{\left[\begin{array}{ll}
0 & 0
\end{array}\right],\left[\begin{array}{l}
0 \\
0
\end{array}\right]\right\} \text {. }
$$

Moreover, consider the sets:

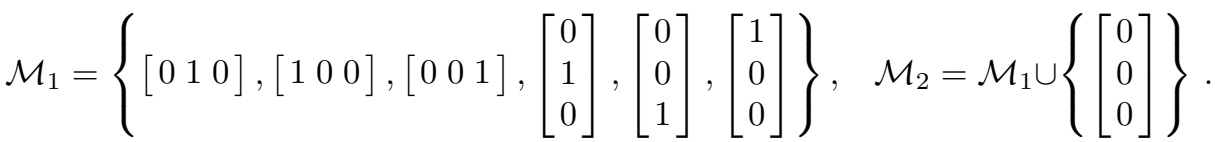

We may easily check that $\mathcal{M}_{1}$ and $\mathcal{M}_{2}$ are antichains (see Fig. 2), and that their avoidance characterizes injections: $\mathcal{I}=A v_{P}\left(\mathcal{M}_{1}\right)=A v_{P}\left(\mathcal{M}_{2}\right)$. So, also $\mathcal{M}_{1}$ and $\mathcal{M}_{2}$ are $m$-bases, although $\mathcal{M}_{1} \subset \mathcal{M}_{2}$.

We recall [3] that the $p$-basis and an $m$-basis of a polyomino class are related by the following.

Proposition 2. Let $\mathcal{C}$ be a polyomino class, and let $\mathcal{M}$ be an $m$-basis of $\mathcal{C}$. Then the $p$-basis of $\mathcal{C}$ consists of all polyominoes that contain a submatrix in $\mathcal{M}$, and that are minimal (w.r.t. $\preccurlyeq P$ ) for this property.

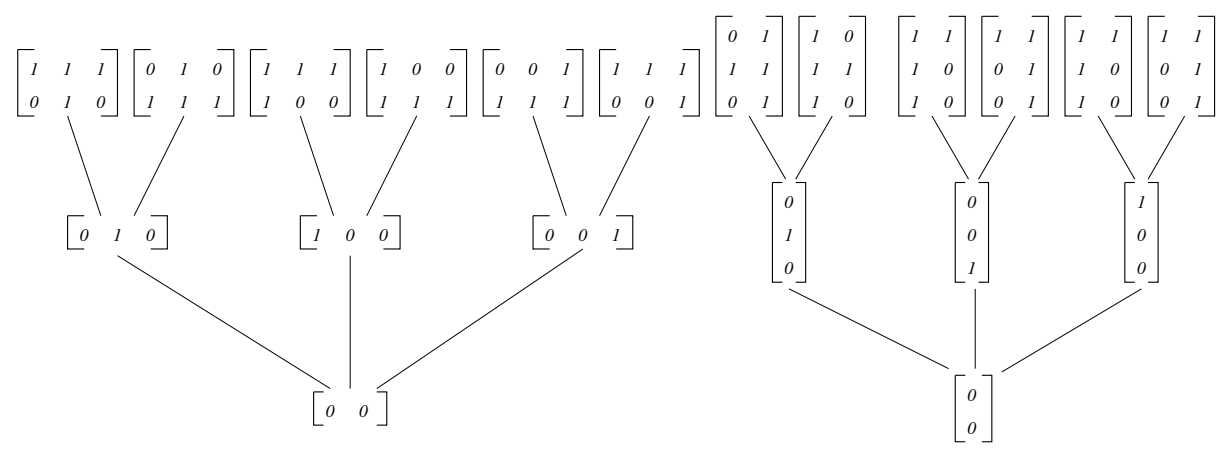

Fig. 2. The $p$-basis and some $m$-bases of $\mathcal{I}$ 
The reader can check the previous property in Fig. 2 for the case of the class $\mathcal{I}$ of injections.

The main goal of this paper is to extend the studies of 34 by adopting a more geometrical approach: we use the notion of pattern avoidance in order to recognize or describe families of polyominoes defined by means of geometrical constraints or combinatorial properties. In particular, we will develop the following research topics:

i) robust polyomino classes, i.e. polyomino classes $\mathcal{C}$ where there is an $m$-basis containing the $p$-basis. We will show that in this case, the $p$-basis is the minimal antichain $\mathcal{M}$ for set inclusion, and for $\preccurlyeq$, such that $A v_{M}(\mathcal{M})=\mathcal{C}$.

ii) given a set of patterns $\mathcal{M}$, study the class of polyominoes avoiding the patterns of $\mathcal{M}$ as submatrices, and give a characterization of this class in terms of the geometrical/combinatorial properties of its elements.

iii) extend the notion of pattern in a polyomino, by introducing generalized polyomino patterns, so that to be able to describe more families of polyominoes known in the literature. Such a generalization resembles what has been done for pattern avoiding permutations with the introduction of vincular, bivincular patterns [5].

For brevity sake, some of the proofs will be omitted. The interested reader can find all the proofs of the paper in Chapter 3 of [2], and the proof of Proposition [10] in the Appendix.

\section{Robust Polyomino Classes}

Every polyomino class is equipped with (at least) two basis, the $p$-basis and the $m$-basis. A natural question is to investigate the relation between the $p$-basis and the $m$-basis, and in particular to understand the conditions that render the $p$-basis the most compact way to describe a polyomino class.

Definition 1. A class is robust when all m-bases contain the p-basis.

The $p$-basis of a robust class has remarkable minimality properties.

Proposition 3. Let $\mathcal{C}$ be a robust class, and let $\mathcal{P}$ be its $p$-basis. Then, $\mathcal{P}$ is the unique $m$-basis $\mathcal{M}$ which satisfies:

(1.) $\mathcal{M}$ is a minimal subset subject to $\mathcal{C}=A v_{P}(\mathcal{M})$, i.e. for every strict subset $\mathcal{M}^{\prime}$ of $\mathcal{M}, \mathcal{C} \neq\left(\mathcal{M}^{\prime}\right)$

(2.) for every submatrix $M^{\prime}$ of some matrix $M \in \mathcal{M}$, we have $M^{\prime}=M$ or $\left.\mathcal{C} \neq A v_{P}\left(\mathcal{M}^{\prime}\right)\right)$, with $\mathcal{M}^{\prime}=\mathcal{M} \backslash\{M\} \cup\left\{M^{\prime}\right\}$.

Proof. Condition (1.) follows directly by Proposition 2, Let us assume that Condition (2.) does not hold, i.e. there exists a proper submatrix $M^{\prime}$ of some matrix $M \in \mathcal{P}$ such that $\mathcal{C}=A v_{P}\left(\mathcal{P}^{\prime}\right)$, with $\mathcal{P}^{\prime}=\mathcal{P} \backslash\{M\} \cup\left\{M^{\prime}\right\}$. So we have that $\mathcal{P}^{\prime} \preccurlyeq_{P} \mathcal{P}$ and $\mathcal{P}^{\prime}$ is an $m$-basis of $\mathcal{C}$. Since $\mathcal{C}$ is a robust class we have that 
$\mathcal{P} \preccurlyeq_{P} \mathcal{P}^{\prime}$ and then $\mathcal{P}=\mathcal{P}^{\prime}$, in particular $M=M^{\prime}$. Suppose that there exists another $m$-basis $\mathcal{M} \neq \mathcal{P}$ satisfying (1.) and (2.). By Proposition 2 , every pattern of $\mathcal{M}$ is contained in some pattern of $\mathcal{P}$, thus $\mathcal{P}$ contains $\mathcal{M}$. Since $\mathcal{C}$ is a robust class, then $\mathcal{P} \subseteq \mathcal{M}$, so $\mathcal{P}=\mathcal{M}$.

We point out that Condition (1.) ensures minimality in the sense of inclusion, while Condition (2.) ensures minimality for $\preccurlyeq$.

Example 2. Let be $\mathcal{C}=A v_{P}\left(P, P^{\prime}\right)$, where $P, P^{\prime}$ are depicted in Figure 3. The class $\mathcal{C}$ is not robust, in fact there is an $m$-basis $M$ disjoint from the $p$-basis:

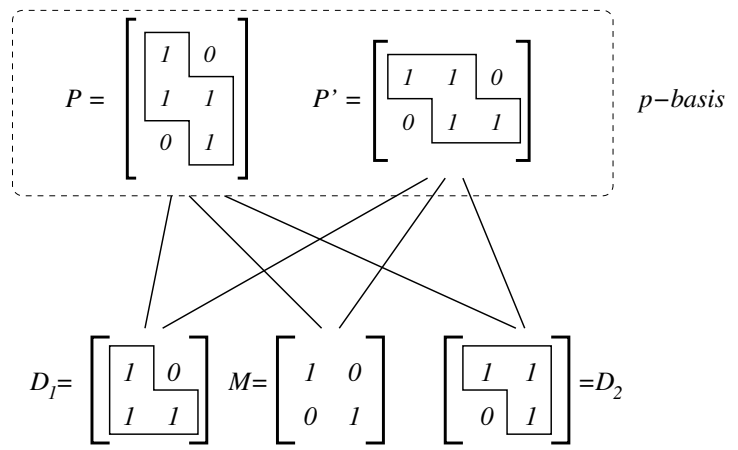

Fig. 3. A non robust class

In practice, $P$ and $P^{\prime}$ are precisely the minimal polyominoes which contain $M$ as a pattern, then by Proposition $2, A v_{P}\left(P, P^{\prime}\right)=A v_{P}(M)$.

In this section, we try to establish some criteria to test the robustness of a class of polyominoes. First, we prove that it is easy to test robustness of a class whose basis is made of just one element:

Proposition 4. Let $M$ be a pattern. Then, $A v_{P}(M)$ is robust if and only if $M$ is a polyomino.

Proof. If $M$ is not a polyomino, then its $p$-basis is clearly different from $P$, so $A v_{P}(M)$ is not robust. On the other side, let us assume that $M$ is a polyomino and that $A v_{P}(M)$ is not robust. Let us assume that an $m$-basis of $A v(M)$ is made of a (non polyomino) matrix $M^{\prime}$ such that $M^{\prime} \preccurlyeq P \quad M$. Since $M^{\prime}$ is not a polyomino then it contains at least two disconnected elements $B$ and $C$, and there are at least two possible ways to connect $B$ and $C$ (by rows or by columns). So, there exists at least another polyomino $P \neq M$ such that $M^{\prime} \preccurlyeq P P$, and $P$ belongs to the $p$-basis of $A v_{P}\left(M^{\prime}\right)$. Thus, $A v_{P}(M) \subseteq A v_{P}\left(M^{\prime}\right)$. The same technique can be used to prove that an $m$-basis of $A v(M)$ canont be made of more than one matrix. 
Now, we aim at extending the previous result to a generic set of polyominoes, i.e. find sufficient and necessary conditions such that, given set of polyominoes $\mathcal{P}$, the class $A v_{P}(\mathcal{P})$ is robust.

Proposition 5. Let be $P_{1}, P_{2}$ two polyominoes and let be $\mathcal{C}=A v_{P}\left(P_{1}, P_{2}\right)$. If for every element $\bar{P}$ in $P_{1} \wedge P_{2}$ we have that:

(1) $\bar{P}$ is a polyomino, or

(2) every chain from $\bar{P}$ to $P_{1}$ (resp. from $\bar{P}$ to $P_{2}$ ) contains at least a polyomino $P^{\prime}$ (resp. $\left.P^{\prime \prime}\right)$, different from $P_{1}$ (resp. $\left.P_{2}\right)$, such that $\bar{P} \preccurlyeq{ }_{P} P^{\prime} \preccurlyeq{ }_{P} P_{1}$ (resp. $\left.\bar{P} \preccurlyeq P P^{\prime \prime} \preccurlyeq P P_{2}\right)$,

then $\mathcal{C}$ is robust.

Proof. Clearly, if $P_{1} \wedge P_{2}$ contains only polyominoes, then $\mathcal{C}$ is robust. On the other side, let $\bar{P} \in P_{1} \wedge P_{2}$, with $\bar{P}$ a non polyomino pattern; then by (2.) every chain from $\bar{P}$ to $P_{1}$ (resp. from $\bar{P}$ to $P_{2}$ ) contains at least a polyomino $P^{\prime}$ (resp. $P^{\prime \prime}$ ), different from $P_{1}$ (resp. $P_{2}$ ). If $\mathcal{C}$ was not robust, $P^{\prime}$ (resp. $P^{\prime \prime}$ ) should belong to the $p$-basis in place of $P_{1}$ (resp. $P_{2}$ ).

Example 3. Let us consider the class $\mathcal{C}=A v_{P}\left(P_{1}, P_{2}\right)$, where $P_{1}$ and $P_{2}$ are the polyominoes depicted in Figure 4. Here, as shown in the picture, $P_{1} \wedge P_{2}$ contains six elements, and four of them are not polyominoes. However, one can check that, for each item $\bar{P}$ of these four matrices, there is a polyomino in the chain from $\bar{P}$ to $P_{1}$ (resp. from $\bar{P}$ to $P_{2}$ ). Thus, by Proposition 5 , the class $\mathcal{C}$ is robust.

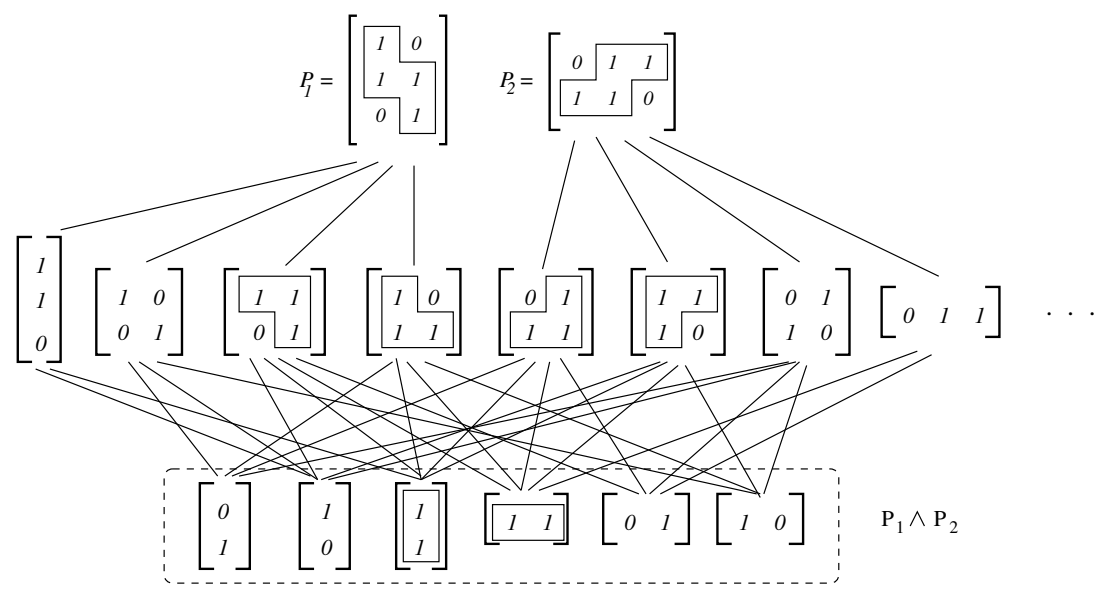

Fig. 4. A robust class 
However, the statement of Proposition 5 cannot be inverted, as we can see in the following example.

Example 4 (Parallelogram polyominoes). We recall that a parallelogram polyomino is a polyomino whose boundary can be decomposed in two paths, the upper and the lower paths, which are made of north and east unit steps and meet only at their starting and final points (see Fig. 5] (c)). We can easily prove that parallelogram polyominoes can be represented by the avoidance of the submatrices:

$$
M_{1}=\left[\begin{array}{ll}
1 & 0 \\
1 & 1
\end{array}\right], M_{2}=\left[\begin{array}{ll}
1 & 1 \\
0 & 1
\end{array}\right] .
$$

These two patterns form a $p$-basis for the class $\mathcal{P}$ of parallelogram polyominoes. Clearly

$$
M_{1} \wedge M_{2}=\left\{\left[\begin{array}{ll}
1 & 1
\end{array}\right],\left[\begin{array}{l}
1 \\
1
\end{array}\right],[0]\right\} .
$$

If $\mathcal{P}$ was not robust, then $M=[0]$ should belong to the an $m$-basis of $\mathcal{P}$; precisely, we should have $A v_{P}(M)=\mathcal{P}$. But this is not true, since clearly $A v_{P}(M)$ is the class of rectangles. Thus, $\mathcal{P}$ is robust. Observe that the set $\left\{M_{1}, M_{2},\left[\begin{array}{lll}1 & 0 & 1\end{array}\right]\right\}$ forms an $m$-basis of the class, but it is not minimal w.r.t. set inclusion.

\section{Classes of Polyominoes Defined by Submatrix Avoidance}

As we have mentioned, several families of polyominoes covered in the literature can be characterized in terms of submatrix avoidance. In particular, if the family of polyominoes is defined by imposing geometric constraints on its elements, then these constraints can be naturally represented by the avoidance of some matrix patterns. For instance, in [4] it was proved that the convexity constraint can be represented by the avoidance of the two submatrices:

$$
H=\left[\begin{array}{lll}
1 & 0 & 1
\end{array}\right] \quad \text { and } \quad V=\left[\begin{array}{l}
1 \\
0 \\
1
\end{array}\right] \text {. }
$$

Similarly, in 3] it was proved that the families of directed-convex, columnconvex, stack polyominoes are polyomino classes. In this section, we consider some polyomino classes which can be represented by the avoidance of submatrices, and deal with the problem of giving a combinatorial/geometrical characterization to these classes. Most of these classes have not been considered yet in the literature, and they show quite simple characterizations and interesting combinatorial properties.

Polyominoes avoiding rectangles. Let $O_{m, n}$ be set of rectangles - binary pictures with all the entries equal to 1 - of dimension $m \times n$ (see Figure 6 $(a)$ ). With $n=m=2$ these objects (also called snake-like polyominoes) have a simple geometrical characterization. 
(a)

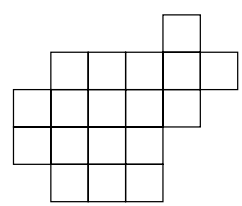

(b)

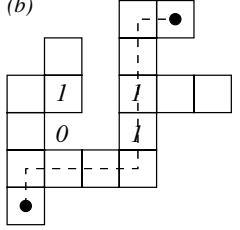

(c)

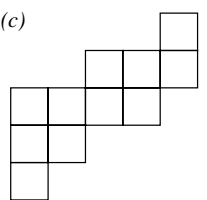

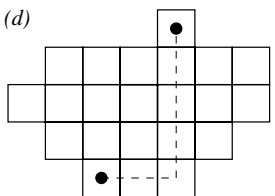

Fig. 5. (a) a convex polyomino; $(b)$ a directed polyomino; $(c)$ a parallelogram polyomino; $(d)$ an $L$-convex polyomino
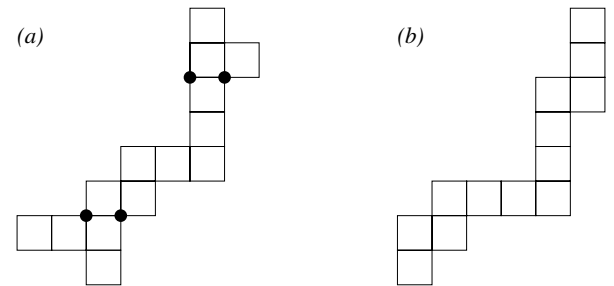

Fig. 6. (a) a snake-like polyomino; $(b)$ a snake

Proposition 6. Every snake-like polyomino can be uniquely decomposed into three parts: a unimodal staircase polyomino oriented with respect to two axisparallel directions $d_{1}$ and $d_{2}$ and two (possibly empty) L-shaped polyominoes placed at the extremities of the staircase. These two L-shaped polyominoes have to be oriented with respect to $d_{1}, d_{2}$.

We have studied the classes $A v_{P}\left(O_{m, n}\right)$, for other values of $m, n$, obtaining similar characterizations which here are omitted for brevity.

Snakes. Let us consider the family of snake-shaped polyominoes (briefly, snakes) - as that shown in Fig. 6 $(b)$ :

Proposition 7. The family of snakes is a polyomino class, which can be described by the avoidance of the following polyomino patterns:
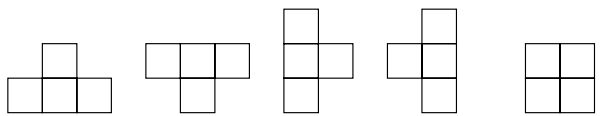

Hollow stacks. Let us recall that a stack polyomino is a convex polyomino containing two adjacent corners of its minimal bounding rectangle (see Fig. $7(a)$ ). Stack polyominoes clearly form a polyomino class, described by the avoidance of the patterns:

$$
\left[\begin{array}{ll}
1 & 1 \\
1 & 0
\end{array}\right] \quad\left[\begin{array}{ll}
1 & 1 \\
0 & 1
\end{array}\right] \quad\left[\begin{array}{lll}
1 & 0 & 1
\end{array}\right]
$$


A hollow stack (polyomino) is a polyomino obtained from a stack polyomino $P$ by removing from $P$ a stack polyomino $P^{\prime}$ which is geometrically contained in $P$ and whose basis lie on the basis of the minimal bounding rectangle of $P$. Figure $7(b),(c)$ depict two hollow stacks.
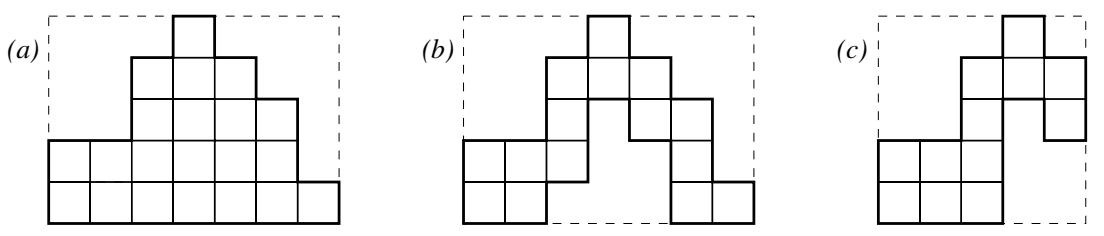

Fig. 7. (a) a stack polyomino; $(b),(c)$ : hollow stacks

Proposition 8. The family $\mathcal{H}$ of hollow stack polyominoes forms a polyomino class with p-basis given by:
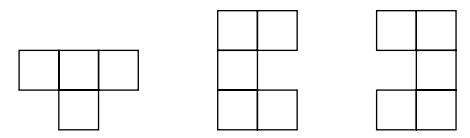

Rectangles with rectangular holes. Let $\mathcal{R}$ be the class of polyominoes obtained from a rectangle by removing sets of cells which have themselves a rectangular shape, and such that there is no more than one connected set of 0's for each row and column. The family $\mathcal{R}$ can easily be proved to be a polyomino class, and moreover:

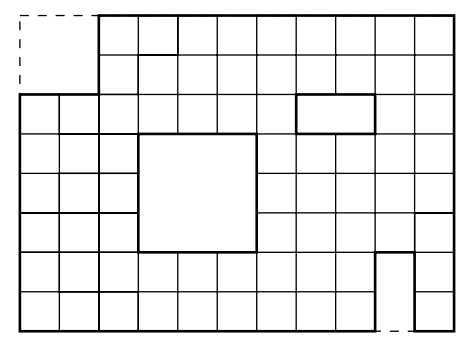

Fig. 8. A rectangle with rectangular holes

Proposition 9. The class $\mathcal{R}$ can be described by the avoidance of the patterns:

$$
\left[\begin{array}{lll}
0 & 1 & 0
\end{array}\right],\left[\begin{array}{l}
0 \\
1 \\
0
\end{array}\right]\left[\begin{array}{ll}
1 & 0 \\
0 & 0
\end{array}\right]\left[\begin{array}{ll}
0 & 1 \\
0 & 0
\end{array}\right]\left[\begin{array}{ll}
0 & 0 \\
1 & 0
\end{array}\right]\left[\begin{array}{ll}
0 & 0 \\
0 & 1
\end{array}\right] \text {. }
$$




\section{Generalized Matrix Patterns}

As already pointed out, there are several families of polyominoes that are not polyomino classes. Amongst them, we have mentioned directed polyominoes and polyominoes without holes. By Proposition 1 these families of polyominoes cannot be expressed in terms of submatrix avoidance. In order to overcome this problem, we extend the notion of pattern in a polyomino, by introducing generalized polyomino patterns, so that to be able to describe more families of polyominoes. Our generalization resembles what has been done for pattern avoiding permutations with the introduction of vincular, bivincular patterns [5].

$L$-convex polyominoes. A convex polyomino is $k$-convex if every pair of its cells can be connected by a monotone path with at most $k$ changes of direction, and $k$ is called the convexity degree of the polyomino 8 . For $k=1$ we have the $L$-convex polyominoes, where any two cells can be connected by a path with at most one change of direction (see Fig. [5 (d)). Recently, $L$-convex polyominoes have been considered from several points of view: in [910] the authors solve the main enumeration problems for $L$-convex polyominoes, while in 7 they approach them from a language-theoretical perspective. In [3] it was shown that $L$-convex polyominoes form a polyomino class, and they can be represented by the avoidance of the submatrices:

$$
H=\left[\begin{array}{lll}
1 & 0 & 1
\end{array}\right], V=\left[\begin{array}{l}
1 \\
0 \\
1
\end{array}\right], S_{1}=\left[\begin{array}{ll}
1 & 0 \\
0 & 1
\end{array}\right], S_{2}=\left[\begin{array}{ll}
0 & 1 \\
1 & 0
\end{array}\right] .
$$

2 -convex polyominoes. Differently from $L$-convex polyominoes, 2 -convex polyominoes do not form a polyomino class. As a matter of fact, the 2-convex polyomino in Figure 9 ( $a$ ) contains the 3 -convex polyomino $(b)$ as a pattern, so the class is not downward closed w.r.t. $\preccurlyeq_{P}$. Similarly, the set of $k$-convex polyominoes is not a polyomino class, for $k \geq 2$.

In practice, this means that 2-convex polyominoes cannot be described in terms of pattern avoidance. In order to be able to represent 2-convex polyominoes we extend the notion of pattern avoidance, introducing the generalized pattern

(a)

\begin{tabular}{|l|ll|l|l|l|}
\hline 0 & 0 & 1 & 1 & 1 \\
\cline { 2 - 5 } & 0 & 0 & 1 & 1 & 0 \\
\cline { 2 - 5 } & 0 & 0 & 1 & 1 & 0 \\
\hline 1 & 1 & 1 & 1 & 0 \\
\hline 1 & 1 & 1 & 0 & 0 \\
\hline
\end{tabular}

(b)

\begin{tabular}{|l|l|l|}
\hline 0 & 1 & 1 \\
\hline 1 & 1 & 0 \\
\hline 1 & 0 & 0 \\
\hline
\end{tabular}

(c)

$\left[\begin{array}{l|ll}0 & 1 & 1 \\ \hline 1 & 1 & 0 \\ 1 & 0 & 0\end{array}\right]$

(d)

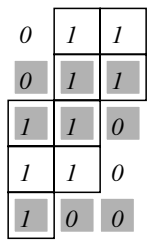

Fig. 9. (a) a 2-convex polyomino $P$; $(b)$ a pattern of $P$ that is not a 2-convex polyomino; $(c)$ a generalized pattern, which is not contained in $(a)$, but is contained in the 3 -convex polyomino (non 2-convex) $(d)$ 
avoidance. Our extension consists in imposing the adjacency of two columns or rows by introducing special symbols, i.e. vertical/horizontal lines: with $A$ being a pattern, a vertical line between two columns of $A, c_{i}$ and $c_{i+1}$ (a horizontal line between two rows $r_{i}$ and $r_{i+1}$ ), will read that $c_{i}$ and $c_{i+1}$ (respectively $r_{i}$ and $r_{i+1}$ ) must be adjacent. When the vertical (resp. horizontal) line is external, it means that the adjacent column (resp. row) of the pattern must touch the minimal bounding rectangle of the polyomino. Moreover, we will use the $*$ symbol to denote 0 or 1 indifferently.

Proposition 10. The class of 2-convex polyominoes can be can be described by the avoidance of the set $\mathcal{M}$ of generalized patterns:

$$
\begin{aligned}
{\left[\begin{array}{lll}
1 & 0 & 1
\end{array}\right]\left[\begin{array}{l}
1 \\
0 \\
1
\end{array}\right] } & {\left[\begin{array}{l|ll}
0 & * & 1 \\
\hline * & 1 & 0 \\
1 & 0 & 0
\end{array}\right]\left[\begin{array}{ll|l}
0 & 0 & 1 \\
0 & 1 & * \\
\hline 1 & * & 0
\end{array}\right]\left[\begin{array}{ll|l}
1 & * & 0 \\
\hline 0 & 1 & * \\
0 & 0 & 1
\end{array}\right] \quad\left[\begin{array}{c|cc}
1 & 0 & 0 \\
* & 1 & 0 \\
\hline 0 & * & 1
\end{array}\right] } \\
& {\left[\begin{array}{cc|cc}
1 & * & 0 & 0 \\
* & 1 & * & 0 \\
\hline 0 & * & 1 & * \\
0 & 0 & * & 1
\end{array}\right] \quad\left[\begin{array}{cc|cc}
0 & 0 & * & 1 \\
0 & * & 1 & * \\
\hline * & 1 & * & 0 \\
1 & * & 0 & 0
\end{array}\right] }
\end{aligned}
$$

The proof of Proposition 10 is reported in the Appendix. Let us just observe, referring to Fig. 9, that the pattern $(c)$ is not contained in the 2-convex polyomino $(a)$, but it is contained in the 3 -convex polyomino $(d)$. It is possible generalize the previous result and give a characterization of the class of $k$-convex polyominoes, with $k>2$, using generalized patterns.

Directed polyominoes. A polyomino $P$ is directed when every cell of $P$ can be reached from a distinguished cell (called the source) by a path - internal to the polyomino - that uses only north and east steps. Figure 5 (b) depicts a directed polyomino. The reader can simply check that the class of the directed polyominoes is not a polyomino class by observing that - in the picture - the four marked cells represent a polyomino which is not directed.

Proposition 11. The class of directed polyominoes can be represented as the class of polyominoes avoiding the following patterns

$$
\left[\begin{array}{l}
1 \\
0
\end{array}\right] \quad\left[\begin{array}{l|l}
0 & 1 \\
\hline * & 0
\end{array}\right]
$$

This proof is analogous to that of Proposition 10, and also relies on the set of patterns determined in [3], whose avoidance describes the class of directedconvex polyominoes. We would like to point out that there are families of polyominoes which cannot be described, even using generalized pattern avoidance. For instance, the reader can easily check that one of these families is that of polyominoes having a square shape. 


\section{References}

1. Barcucci, E., Frosini, A., Rinaldi, S.: On directed-convex polyominoes in a rectangle. Discrete Math. 298(1-3), 62-78 (2005)

2. Battaglino, D.: Enumeration of polyominoes defined in terms of pattern avoidance or convexity constraints, PhD thesis (University of Sienna), arXiv:1405.3146v1 (2014)

3. Battaglino, D., Bouvel, M., Frosini, A., Rinaldi, S.: Permutation classes and polyomino classes with excluded submatrices. ArXiv 1402.2260 (2014)

4. Battaglino, D., Bouvel, M., Frosini, A., Rinaldi, S., Socci, S., Vuillon, L.: Pattern avoiding polyominoes. In: Proceedings di Italian Conference Theoretical Computer Science, Palermo, Settembre 9-11 (2013)

5. Bona, M.: Combinatorics of permutations. Chapman-Hall and CRC Press (2004)

6. Brändén, P., Claesson, A.: Mesh patterns and the expansion of permutation statistics as sums of permutation patterns. Electr. J. of Combin. 18(2) (2011) (The Zeilberger Festschrift volume)

7. Brocchi, S., Frosini, A., Pinzani, R., Rinaldi, S.: A tiling system for the class of L-convex polyominoes. Theor. Comput. Sci. 457, 73-81 (2013)

8. Castiglione, G., Restivo, A.: Ordering and Convex Polyominoes. In: Margenstern, M. (ed.) MCU 2004. LNCS, vol. 3354, pp. 128-139. Springer, Heidelberg (2005)

9. Castiglione, G., Frosini, A., Munarini, E., Restivo, A., Rinaldi, S.: Combinatorial aspects of $L$-convex polyominoes. European J. Combin. 28, 1724-1741 (2007)

10. Castiglione, G., Frosini, A., Restivo, A., Rinaldi, S.: Enumeration of $L$-convex polyominoes by rows and columns. Theor. Comput. Sci. 347, 336-352 (2005)

11. Klazar, M.: On abab-free and abba-free sets partitions. European J. Combin. 17, 53-68 (1996)

12. Knuth, D.E.: The Art of Computer Programming, 2nd edn. Volume 1: Fundamental algorithms; Addison-Wesley Series in Computer Science and Information Processing. Addison-Wesley Publishing Co., Reading (1975)

13. Rowland, E.: Pattern avoidance in binary trees. J. Combin. Theory Ser. A 117, 741-758 (2010)

14. Sagan, B.E.: Pattern avoidance in set partitions. Ars Combin. 94, 79-96 (2010) 


\section{Appendix}

Proof of Proposition 10. We recall that in a 2-convex polyomino, for each two cells, there is a path connecting them, which uses only two types of steps among $n, s, e, w$ (north, south, east and west unit steps, respectively) and has at most two changes of direction. Moreover, for any two cells $c_{1}$ and $c_{2}$ of a polyomino, the minimal number of changes of direction from $c_{1}$ to $c_{2}$ can be computed from just two paths, starting with a vertical and a horizontal step, respectively, in which every side has maximal length. We will refer to these as the extremal paths connecting $c_{1}$ and $c_{2}$.

$(\Rightarrow)$ If $P$ is a 2-convex polyomino then $P$ avoids $\mathcal{M}$.

Let us assume by contradiction that $P$ is a 2-convex polyomino containing one of the patterns of $\mathcal{M}$, clearly not $H$ and $V$, by convexity. For simplicity sake, we will consider only the two patterns of $\mathcal{M}$,

$$
Z_{1}=\left[\begin{array}{c|cc}
0 & * & 1 \\
* & 1 & 0 \\
1 & 0 & 0
\end{array}\right] \text { and } Z_{2}=\left[\begin{array}{cc|cc}
0 & 0 & * & 1 \\
0 & * & 1 & * \\
\hline * & 1 & * & 0 \\
1 & * & 0 & 0
\end{array}\right]
$$

since the proof for the other patterns can be obtained by symmetry. If $P$ contains $Z_{1}$ (resp. $Z_{2}$ ) then it has to contain a submatrix $P^{\prime}$ (resp. $P^{\prime \prime}$ ) of the form:

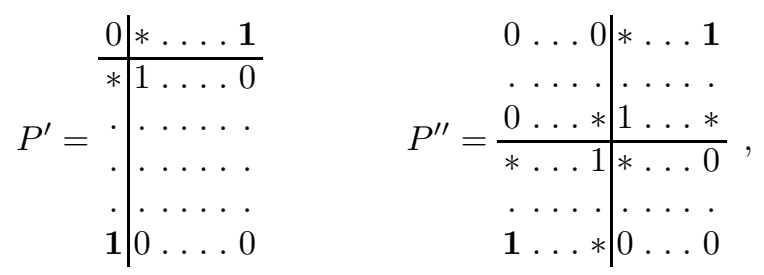

where the $0,1, *$ are the elements of $Z_{1}$ (resp. $Z_{2}$ ) and the dots can be replaced by 0,1 indifferently, clearly in agreement with the convexity and polyomino constraints.

Among all the polyominoes which can be obtained from $P^{\prime}$ (resp. $P^{\prime \prime}$ ), the one having the minimal convexity degree is that, called $\bar{P}^{\prime}$ (resp. $\bar{P}^{\prime \prime}$ ), having the maximal number of 1 entries. It is easy to verify that the minimal number of changes of direction requested to connect the 1 entries in boldface of $\bar{P}^{\prime}$ (resp. $\bar{P}^{\prime \prime}$ ) is three, so $\overline{P^{\prime}}$ (resp. $\bar{P}^{\prime \prime}$ ) is a 3 -convex polyomino, which contradicts our assumption.

$(\Leftarrow)$ If $P$ avoids $\mathcal{M}$ then $P$ is a 2 -convex polyomino.

Again by contradiction let us assume that $P$ avoids $\mathcal{M}$ and it is a 3 -convex polyomino, i.e. there exist two cells of $P, c_{1}$ and $c_{2}$, such that any path from $c_{1}$ to $c_{2}$ requires at least three changes of direction.

Let us take into consideration the two extremal paths from $c_{1}$ to $c_{2}$. The only possible cases are the following (up to rotation):

- the two extremal paths are distinct, Fig. 10 (a); 
- one of the extremal paths does not exist, see Fig. 10 (b);

- the two extremal paths coincide after the first change of direction, see Fig. 10 (c);

- the two extremal paths coincide after the second change of direction, see Fig. 10 $(d)$.

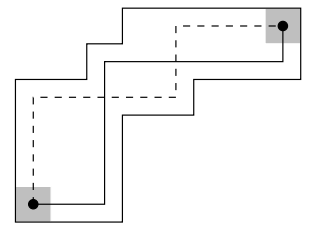

(a)

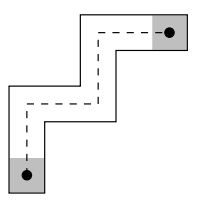

(b)

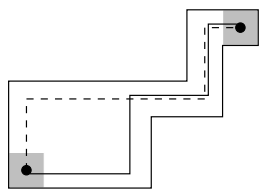

(c)

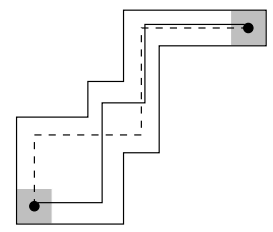

(d)

Fig. 10. The possible cases of extremal paths connecting the cells $c_{1}$ and $c_{2}$

Here, we will consider only the first case, since the others are abalogous: as sketched in the picture below, the polyomino $P$ of Fig. 10 (a) has to contain a submatrix $P^{\prime}$ - given by the boldface entries - of the form:

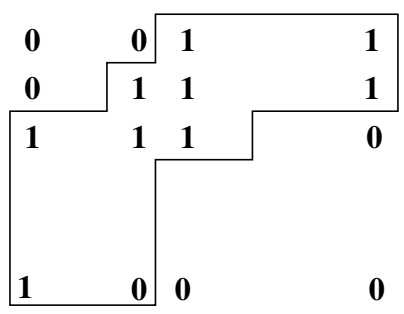

It is easy to see that such a submatrix is one of those that we can obtain replacing appropriately the symbol $*$ in the pattern $Z_{2}$. So, $P$ contains $Z_{2}$ against the hypothesis. We point out that the pattern $Z_{1}$, and its rotations, can be obtained from the pattern $Z_{2}$ (or its rotation) replacing appropriately the $*$ entries, but we need to consider them in order to include the 3 -convex polyominoes with three rows or columns. 